\title{
MAGNETOOPTICAL STUDIES OF CdTe-RICH EPITAXIAL LAYERS
}

\author{
E. Dudziak, J. Bożym, D. Pruchnik and L.Z. Jędral \\ Institute of Physics, Technical University of Wroclaw \\ Wybrzeże Wyspiańskiego 27, 50-370 Wrocław, Poland
}

The first magnetooptical studies comprising magnetoreflection, magnetoabsorption and Faraday rotation of CdTe-rich $\mathrm{Hg}_{1-x-y} \mathrm{Cd}_{x} \mathrm{Mn}_{y} \mathrm{Te}$ are reported. The drastic disappearing of excitonic structure, for already very small $\mathrm{Hg}$ content, has been observed. The exchange parameters close to those for CdMnTe have been found.

PACS numbers: $71.25 . \mathrm{Tn}, 72.40 .+\mathrm{w}, 78.20 . \mathrm{Ls}$

\section{Introduction}

HgTe-rich $\mathrm{Hg}_{1-x-y} \mathrm{Cd}_{x} \mathrm{Mn}_{y} \mathrm{Te}$ (HCMT) alloys have recently attracted much attention as a new candidate for infrared detectors operating in spectral range of 5-15 $\mu \mathrm{m}[1,2]$. Much less work has been done on CdTe-rich side of the alloys although this wide-gap material shows also a promise of high-performance photodetectors for wavelengths of 1.3-1.8 $\mu \mathrm{m}$ [3]. HCMT is a semimagnetic semiconductor giving the opportunity to change the energy gap by varying the CdTe composition independently of the MnTe content. This paper reports the first magnetooptical studies comprising magnetoreflection, magnetoabsorption and Faraday rotation of CdTe-rich HCMT in the fundamental absorption region.

\section{Experiment}

The measurements were performed on graded-gap epitaxial HCMT layers, about $200 \mu \mathrm{m}$ thick, grown by isothermal vapour phase epitaxy. CdMnTe wafers with $5 \%-\mathrm{MnTe}$ content were used as a substrate.

The measurements of magnetoreflection, magnetoabsorption and Faraday rotation in the fundamental absorption region were performed on samples etched to a given surface molar composition. The samples were placed in the superconducting solenoid with magnetic field up to $6 \mathrm{~T}$ and the temperature of the samples was lowered down to $7 \mathrm{~K}$. 


\section{Results and discussion}

The measured magnetoreflectivity spectra of CdTe-rich HCMT with very low $\mathrm{Hg}$ concentration $(<0.001)$ showed a typical splitting of the free exciton line into four components. In a simple exchange interaction model [4] $\alpha / \beta$ and $N_{0}(\alpha-\beta)$ were determined from the energy differences between the $\sigma^{+}$and $\sigma^{-}$weak and strong components ( $N_{0}$ is the number of cations per unit volume, $\alpha$ and $\beta$ are the exchange integrals for conduction and valence band, respectively). The average spin component $\left\langle S_{z}\right\rangle$-was taken from [5].

For a little higher $\mathrm{Hg}$ concentration $(>0.001)$ a drastic disappearing of the excitonic structure in the reflectivity spectra was observed. An application of photomodulated technique did not reveal any structure either, though for $\mathrm{HgCdTe}$ (HCT) epitaxial layers [6] excitonic features in reflectivity spectra were observed up to $20 \%$ of $\mathrm{Hg}$ molar composition. In our opinion this difference results from higher than in HCT concentrations of free carriers which effectively decrease the hole-electron interaction.

For samples with no exciton structure in reflectivity spectra, the shift of the fundamental absorption edge with magnetic field was studied (Fig. 1). The

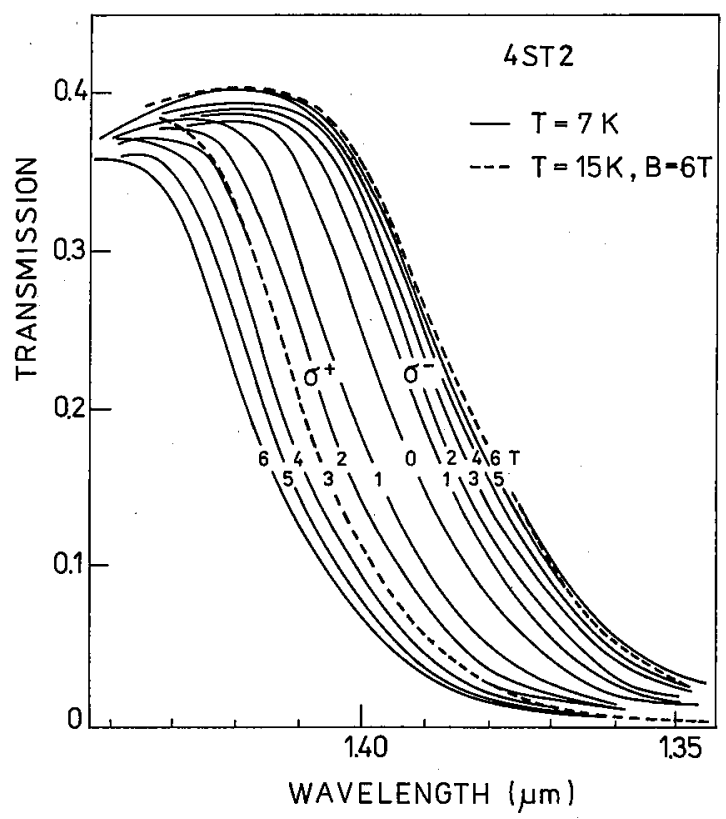

Fig. 1. Shift of the fundamental absorption edge in magnetic field for $\sigma^{+}$and $\sigma^{-}$light polarization. Sample 4ST2 with surface composition: $x=0.59$ and $y=0.028$.

absorption edge energy $E_{0}$ at constant transmission coefficient ( 0.05$)$ was assumed as an "energy gap" [6]. There is a clear asymmetry in the $E_{0}$ shift with magnetic field for $\sigma^{+}$and $\sigma^{-}$polarizations. A similar effect has been observed in $\mathrm{CdMnTe}$ 
[7] where the $\sigma^{-}$edge has moved towards higher energy in low magnetic fields and then with the increasing magnetic field it has moved back, towards lower energies. This strange behaviour has been explained in [8] as a consequence of the mixing between states allowed in the $\sigma^{+}$and $\sigma^{-}$polarizations. Transitions to excited exciton states allowed in $\sigma^{+}$polarization are also allowed and even much stronger in $\sigma^{-}$polarization and determine the $\sigma^{-}$edge. In the case of HCMT samples this effect is weaker, probably due to the mentioned disappearing of the excitonic structure. The shift of the non-disturbed $\sigma^{+}$edge from the position in the absence of magnetic field was taken as a measure of spin splitting.

Since in the case of our composition-graded thin layers direct magnetization measurements were impossible the Faraday rotation in the region of the fundamental absorption edge was used as a measure of a magnetization $[9,10]$. Oscillations of a giant Faraday rotation were observed. Fitting procedure of the formula from

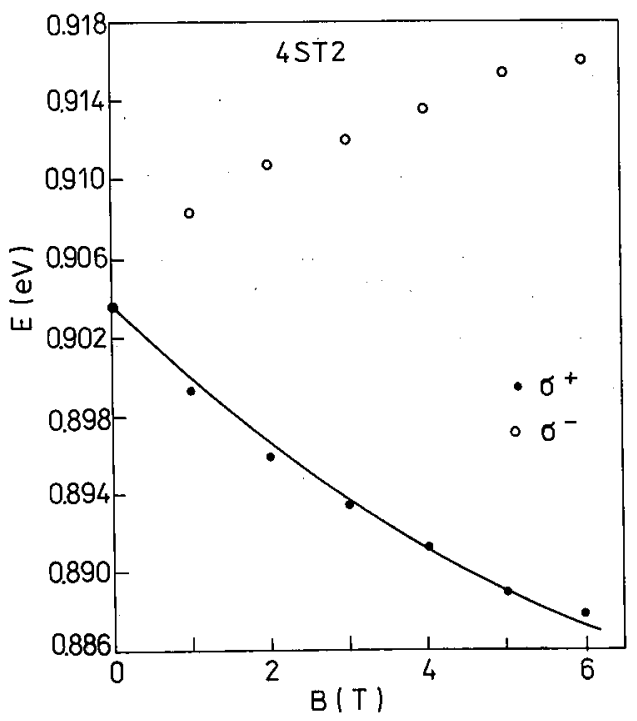

Fig. 2. Absorption edge energies $E_{0}$ (see text) vs. magnetic field for $\sigma^{+}$and $\sigma^{-}$ polarization. Solid line - the theory with $N_{0}(\alpha-\beta)=1.0 \mathrm{eV}$.

[5] to our experimental Faraday rotation results gave us the parameter $T_{0}$ in the modified Brillouin function for $\left\langle S_{z}\right\rangle$ (Fig. 2).

The analysis of the current results as well as our previous results [2] for narrow-gap HCMT allow us to conclude that within the experimental error limits the exchange parameters for HCMT are close to those obtained for CdMnTe:

$$
\begin{aligned}
& \alpha / \beta=-0.25 \pm 0.05, \\
& N_{0}(\alpha-\beta)=(1.1 \pm 0.1) \mathrm{eV}
\end{aligned}
$$




\section{Acknowledgements}

The authors wish to thank Professor J.A. Gaj for the helpful discussion.

\section{References}

[1] S. Wong, P.Becla, J. Vac. Sci. Technol. A 4, 2019 (1986).

[2] E. Dudziak, L.Z. Jędral, E. Płaczek-Popko, J. Bożym, J.F. Kasprzak, Acta Phys. Pol. A77, 171 (1990).

[3] S.H. Shin, J.G. Pasko, P.S. Lo, W.E. Tennat, J.R. Anderson, M. Górska, M.Fotouhi, C.R.Lu, Mat. Res. Symp. Proc. 89, 267 (1987).

[4] J.A. Gaj, J. Ginter, R.R. Gałazka, Phys. Status Solidi B 89, 655 (1978).

[5] N. Yamada, S. Takeyama, T. Sakakibara, T. Goto, N. Miiura, Phys. Rev. $B$ 34, 4121 (1986).

[6] J. Camassel, J.P. Laurenti, A. Bouhemadou, R. Legros, A.Lusson, Phys. Rev. B 38, 3948 (1988).

[7] M.Z. Cieplak, P. Byszewski, Solid State Commun. 29, 81 (1979).

[8] Nguyen The Khoi, J. Ginter, A. Twardowski, Phys. Status Solidi B 117, 67 (1983).

[9] J.A. Gaj, in Semiconductors and Semimetals, Ed. J.K. Furdyna, J. Kossut, Vol. 25, Academic Press, San Diego 1988, p. 275.

[10] D. Heiman, F.D. Isaacs, P. Becla, S. Foner, Phys.Rev. B 35, 3307 (1987). 